\title{
Coronary Fistulous Communications with Left Ventricular Chamber and Giant Mural Thrombus after Myocardial Infarction
}

\author{
Sugato NawA, M.D., Hiroyuki Soga, M.D., \\ Atsushi Urakami, M.D., Yasuo Mryachi, M.D.,* \\ Norihide Toshino, M.D., * Kenji HaYashi, M.D., * \\ and Nobuyoshi Shrmizu, M.D.
}

\begin{abstract}
SUMMARY
Selective coronary arteriography, performed in a 67-year-old man with post-infarction angina, demonstrated severe three vessel disease and coronary fistulous communications with the left ventricular chamber adjacent to a giant mural thrombus formed in the apical aneurysm. Most of the contrast media seemed to empty directly into the chamber without presenting hypervascular blushes of the thrombus itself. This was somewhat different from the observation previously reported in a case with coronary fistulae associated with a post infarction mural thrombus. It was stressed that one should not misinterpret this condition as a rare coronary artery-cardiac chamber shunt associating myocardial infarction. (Jpn Heart J 35: 801-808, 1994)
\end{abstract}

Key words: Coronary fistula Mural thrombus Myocardial infarction

CORONARY artery-cardiac chamber shunts are still not frequently 4 observed even after the widespread use of selective coronary arteriography. ${ }^{1-3)}$ On the other hand, it is known that neovascularity with fistulous communications with the cardiac chambers will sometimes develop in a mural thrombus after myocardial infarction (MI). ${ }^{3-6)}$ This condition, however, has sometimes been erroneously reported as a rare type of coronary fistula associating MI. ${ }^{1,7,8)}$ Therefore, this should be kept in mind when establishing the correct diagnosis. This paper reports a case of coronary fistulae associated with left ventricular mural thrombus after MI, where the angiographic features were somewhat different from those previously described. ${ }^{3-6)}$

From the Second Department of Surgery, Okayama University Medical School, Okayama, and JunkankikaHayashi Hospital, Nihama, Japan.

Address for correspondence: Sugato Nawa, M.D., The Second Department of Surgery, Okayama University Medical School, 2-5-1 Shikata-cho, Okayama 700, Japan.

Received for publication June 6, 1994.

Accepted September 2, 1994. 


\section{Case Report}

A 67-year-old man with a history of inferior MI in 1980 and anteroseptal MI in 1983 was referred on September 25, 1989 for investigation of precordial pain which had recently increased in frequency. There were no risk factors except smoking, which had been discontinued after the first MI.

He had a normal sinus rhythm with a blood pressure of $146 / 78 \mathrm{mmHg}$; triplets of ventricular premature contractions were noted on 24-hour dynamic electrocardiogram. On auscultation, no heart murmur was heard, and breath sounds were normal. The electrocardiogram recorded on referral showed evidence of old inferior and anteroseptal MIs (Figure 1). Chest x-ray examination showed a normal cardiac silhouette with a cardiothoracic ratio of $46.4 \%$. Echocardiographic study revealed a large aneurysm formed at the left ventricular apex, where a giant mural thrombus of more than $5 \mathrm{~cm}$ (maximal length) was present. Thallium-201 myocardial perfusion imagings at rest showed hypoaccumulation in the site corresponding to the infarcted area (Figure 2), and the left ventricular ejection fraction was estimated to be $40.9 \%$ by gated pool scintigraphy.

Cardiac catheterization including left ventriculography and coronary arteriography was done. The left ventriculogram confirmed a large apical aneurysm, the anteroseptal wall of which was faintly calcified (arrow) (Figures 3). As observed on echocardiograms, a large apical filling defect, indicating a mural thrombus, was also demonstrated. The left ventricular segmental wall motion showed hypokinesis, akinesis, and dyskinesis of segments 2 and 5, segment 4 , and segment 3 , respectively, on cine-ventriculograms. The right coro-

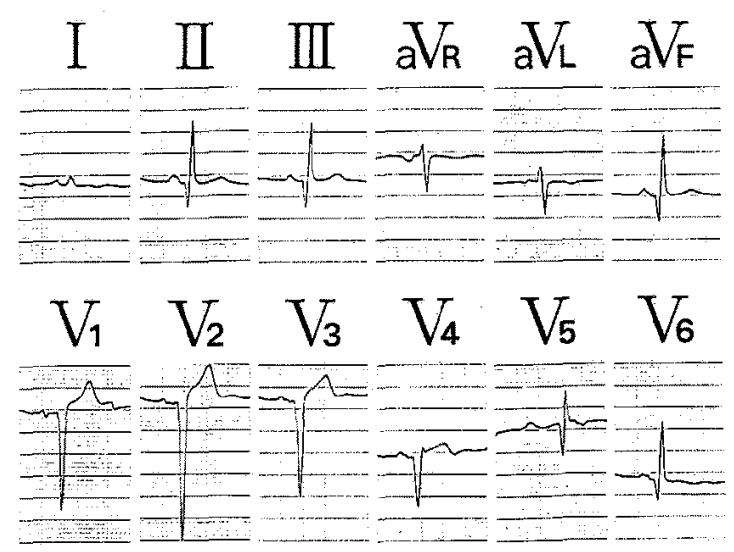

Figure 1. Electrocardiogram recorded on referral. Convincing $Q$ waves are seen in leads II, III, $\mathbf{a} V_{F}$, and $V_{1}$ through $V_{6}$. 


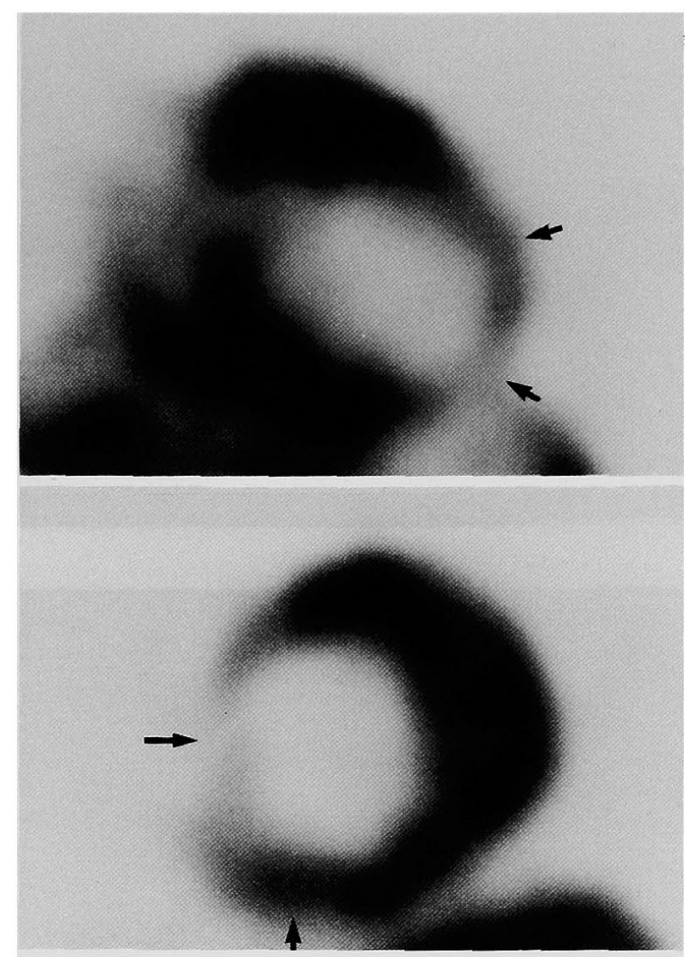

Figure 2. Thallium-201 myocardial perfusion imagings. Top: Anteroposterior projection. Severe low perfusion is noted at the anteroapical site (arrows), while that at the inferior wall is not clearly demonstrated. Bottom: Left anterior oblique (60 degree) projection. The anteroseptal site (long arrow) is little perfused, and the site representing the apex and inferior wall (short arrow) shows relatively low perfusion also.

nary artery was completely obstructed distally to the origin of the second right ventricular branch, and the distal parts were visualized by a maze of collaterals arising chiefly from the right ventricular branches (Figure 4-A). At the same time, the left anterior descending artery (LAD) and diagonal branch were faintly opacified in a retrograde fashion via the septal branches (SEPTs). No fistula from the right coronary artery was visualized. Left coronary arteriography revealed that the LAD was completely occluded at the take-off of the diagonal branch, and that the circumflex artery was also obstructed, while only the high lateral branch appeared normal (Figure 4-B). The distal branches of the LAD, SEPTs in particular, were visualized through the labyrinth of collaterals chiefly from the diagonal branch in association with the intramural bridge bypass. Interestingly, multiple fistulae were observed through which the contrast media drained into the left ventricular chamber (Figure 4-B), presenting smoking signs. ${ }^{3)}$ The fistulae were found adjacent to the mural thrombus, and it seemed that the fistulous 
$\mathbf{A}$

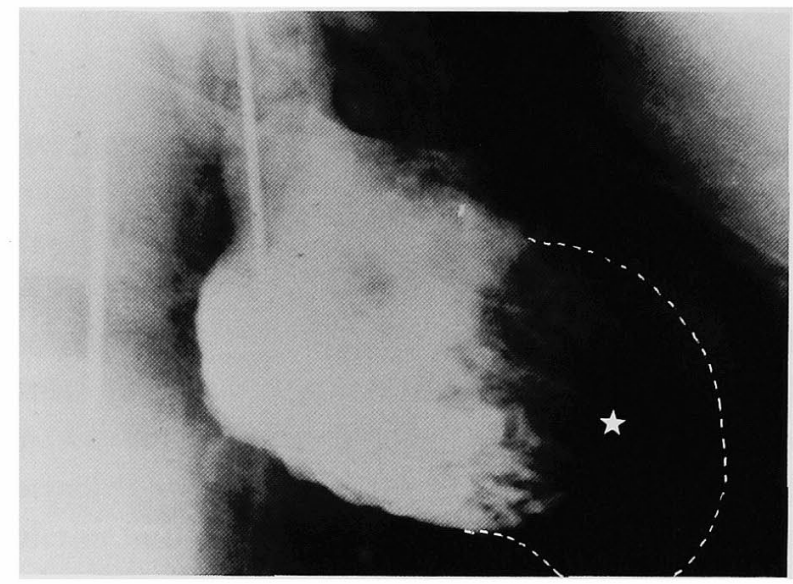

B

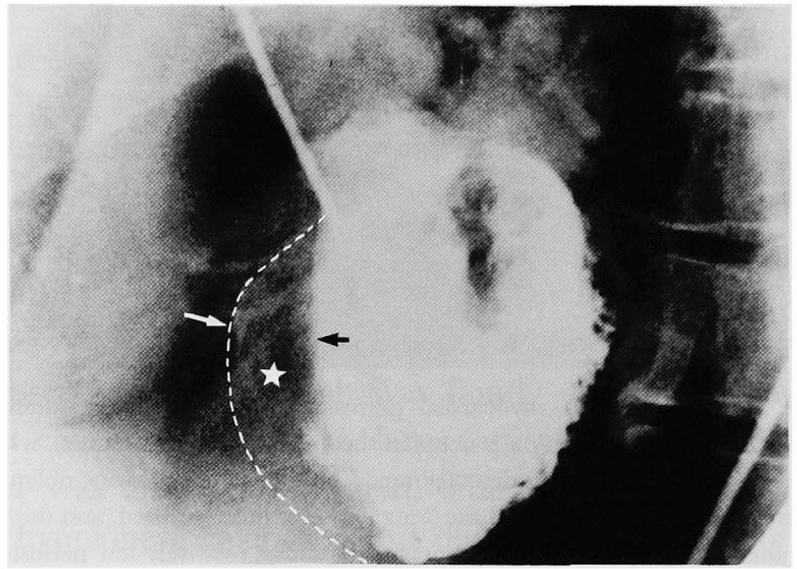

Figure 3. Left ventriculograms. A: Right anterior oblique (40 degree) projection. An apical aneurysm (dotted line) is observed. The large filling defect of contrast media (star) indicates the presence of a giant mural thrombus. B: Left anterior oblique (60 degree) projection. The long arrow indicates the faint calcification of a septal part of the aneurysm (dotted line), which is seen to protrude more laterally, in this view, than the septal margin (short arrow). A filling defect of contrast media (star), indicating mural thrombus, is seen in this view also.

orifices were located at the marginal site of the thrombus. Not only the septal branches but also the diagonal branch was seen forming these fistulae. Importantly, it seemed not to be the mural thrombus but rather the infarcted myocardium that presented hypervascular blushes. That is, most of the contrast media seemed to escape directly from small vessels in the infarcted wall into the ventricular chamber without staining the mural thrombus. The contrast material was seen to stagnate in the sulci of the thrombus and then to diffuse slowly into the chamber, outlining the thrombus (Figure 4-b). The native right and left coronary arteries were neither dilated nor tortuous at any site, and this was the case with 
$\mathbf{A}$

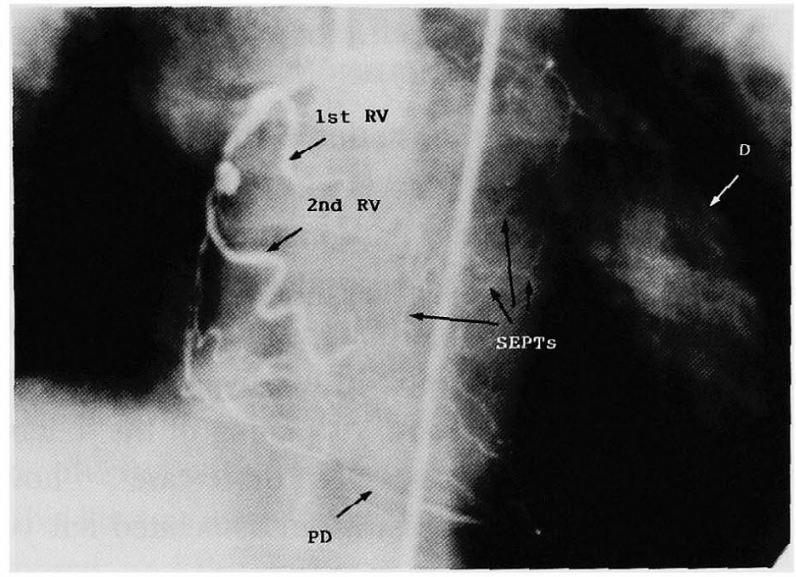

B

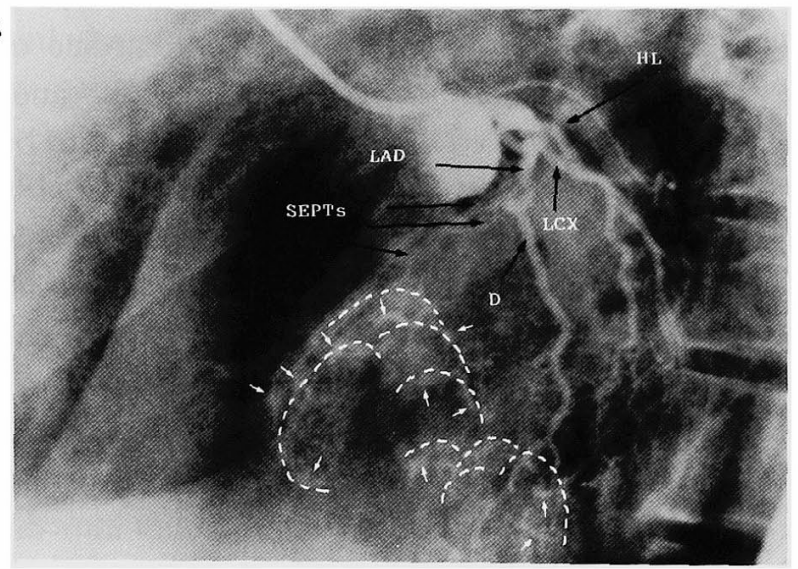

Figure 4. Coronary arteriograms. A: Right coronary arteriogram in the right anterior oblique ( 40 degree) projection. Abbreviations: 1 st $\mathrm{RV}=$ the first right ventricular branch; 2nd $\mathrm{RV}=$ the second right ventricular branch; $\mathrm{PD}=$ posterior descending artery; SEPTs = septal branches; $\mathrm{D}=$ diagonal branch. B: Left coronary arteriogram in the left anterior oblique (60 degree) projection. Smoking signs are indicated with small short arrows, and the outline of the giant mural thrombus of an irregular shape with dotted lines. The findings in this projection correlate well with those in Figure 3B. Abbreviations: $\mathrm{LAD}=$ left anterior descending artery; $\mathrm{LCX}=$ left circumflex artery; $\mathrm{HL}=$ high latcral branch. Other abbreviations are the same as in Figure 4-A.

the arterial branches forming fistulae.

Surgical intervention was refused, and the patient received medical treatment. Unfortunately, however, the patient died of a cerebrovascular accident 2 years later at another hospital; we were not given the details. 


\section{Discussion}

Coronary artery fistulae communicating with left cardiac chambers are generally considered uncommon. The association of a coronary fistula of this type with $\mathrm{MI}$ is thought to be very rare. It has long been debated, however, whether the association of these two conditions is incidental or causal. In fact, a few papers reported the development of coronary fistulae after MI. ${ }^{78)}$ With respect to its mechanism, Ryan and Gertz supported the hypothesis suggested by Phillips and Libanoff; extensive collateralization, reopening of the Thebesian veins, and communications between the two systems. ${ }^{87}$ In those cases, ${ }^{7,8)}$ however, it was not clearly described whether or not there was an associated left ventricular mural thrombus.

On the other hand, we should comment on another type of fistula, that is, neovascularity having fistulous communications with cardiac chambers, which developed in a mural thrombus formed in a left ventricular aneurysm after MI. This should be more often observed but seems occasionally to be misinterpreted as the aforementioned rare coronary fistulae. ${ }^{1,7,8)}$ The abnormal vascularity in the left ventricular mural thrombus was first demonstrated angiographically by Grollman et al. ${ }^{4}$ Soulen et al considered the coronary neovascularity and fistula formation as a sign of mural thrombus with histological evidence. ${ }^{5)}$ Tomotsune et al have also reported a detailed evaluation of 9 such cases, where they expressed the neovascularity in terms of "a dense mass of small vessels resembling a toothbrush." ${ }^{.6)}$ More recently, Matsunaga has clearly distinguished this one by classifying the coronary artery-cardiac chamber shunts into three types. ${ }^{3)}$ The characteristic features reported ${ }^{3-6)}$ are summarized as; 1 ) neovascular labyrinth or a small dense mass of new vessels presenting hypervascular blushes within the thrombus or chamber filling defects, 2) washout of the contrast material into the cardiac chambers (smoking sign) after blushing; absence of a venous system, 3) no dilation of the native coronary arteries having fistulous communications, 4) predilection for left cardiac chambers, 5) development in a relatively fresh thrombus, 6) absence of hemodynamically significant shunts. Although the findings in the present case met the majority of these features, the most marked difference was that it was not the thrombus itself but rather the ventricular wall that presented hypervascular blushes. That is, most of the contrast media seemed to empty directly into the ventricular chamber without staining the thrombus. Indeed, blushes were observed at the site of the mural thrombus, but with careful observation it seemed that the blushes were not "within" the thrombus but rather in the sulci on the surface of the thrombus, stagnating there and then slowly diffusing into the chamber, outlining the thrombus. Besides, such fistulous communications were found chiefly at the thrombus margin neighboring the ventricular wall. 
These were remarkably different from the observations reported; at least, it was unlike "a dense mass of small vessels". ${ }^{6}$ ) We have no histological evidence, but we consider the mechanism of presenting unique angiographic features in this case as follows. With respect to the mechanism of development of neovascularity in a mural thrombus after MI, we agree with Soulen R1, et al..$^{5}$ and Tomotsune $\mathrm{K}$, et al. ${ }^{6)}$ But, additionally, we believe that the aforementioned mechanism proposed by Phillips and Libanoff ${ }^{\text {b) }}$ will also participate in developing neovascularity in a mural thrombus. Autolysis of the thrombus, with its feeding blood channels left open, was considered with regards to the development of fistulous communications of neovascularity with a cardiac chamber. ${ }^{4,57}$ Accordingly, when thrombolysis of this kind occurred so that the original endothelial surface of the ventricle was exposed, and this occurred at the peripheral site of the thrombus, the coronary artery system was able to communicate directly with a cardiac chamber. We think the reason why the thrombus itself was not clearly stained is as follows. Many papers ${ }^{3-6)}$ have reported that neovascularity was usually observed in relatively fresh thrombi, and that the mural thrombus is gradually organized and blood channels are obliterated as time passes, resulting in a loss of blushes of the thrombus. We think this to be true in the present case, since about 6 years had passed after the anteroseptal MI, though we unfortunately could not estimate the age of the thrombus. The unique coronary arteriographic features in the present case were thus derived in our opinion.

This paper reports a case of coronary fistulous communications with the left ventricular chamber and giant mural thrombus after MI that presented unique angiographic features; it is stressed that this type of coronary artery fistulae should not be misinterpreted as a rare coronary artery-cardiac chamber fistula associating MI.

\section{REFERENCES}

1. Arani DT, Greene DG, Klocke FJ: Coronary artery fistulas emptying into left heart chambers. Circulation 54 (Suppl II): II $\cdots 39,1976$

2. Kono J, Okubo S, Yamada H, Hongo M, Misawa T, Iwanami S, Kinoshita O, Kusama S: Clinical study of 12 cases with coronary artery fistula. Heart 20:1281, 1988 (in Japanese)

3. Matsunaga N: Radiological evaluation of coronary artery cardiac chamber shunt. J Jpn Assoc Med Radiol 47: 1170, 1987 (in Japanese)

4. Grollman JH Jr, Hoffman RB, Price JE Jr, O'Reilley RJ, Lilley JM, Herman NP: Abnormal vascular. ity in left ventricular mural thrombus demonstrated by selective coronary arteriography. Radiology 113 : 591,1974

5. Soulen RL, Grollman JH Jr, Paglia D, Kreulen T: Coronary neo-vascularity and fistula formation. A sign of mural thrombus. Girculation 56: 663,1977

6. Tomotsune K, Shimizu M, Wakiya Y, Sakurai H, Kanoh T, Okada R, Kitamura K: Coronary neovascularity related to a left ventricular mural thrombus. Jpn Heart J 24: 325, 1983

7. Maeda T, Hiasa Y, Iwano K, Ishida T, Aihara T, Bando M, Nakai Y, Kataoka Y, Mori H: A case of left coronary artery fistula opening to left ventricle with myocardial infarction. Intern Med 52: 1163, 1983 (in Japanese) 
8. Ryan $\mathrm{G}$, Gertz EW: Fistula from coronary arteries to left ventricle after myocardial infarction. $\mathrm{Br}$ Heart J 39: 1147, 1977 